\title{
Detection of membrane fluidity in submitochondrial particles of platelets and erythrocyte membranes from Mexican patients with Alzheimer disease by intramolecular excimer formation of 1,3 dipyrenylpropane
}

\author{
G.G. Ortiz ${ }^{\mathrm{a}, *}$, F. Pacheco-Moisés ${ }^{\mathrm{b}}$, M. El Hafidic ${ }^{\mathrm{c}}$, A. Jiménez-Delgado ${ }^{\mathrm{b}}$, M.A. Macías-Islas ${ }^{\mathrm{d}}$, \\ S.A. Rosales Corral ${ }^{\mathrm{a}}$, A. Célis de la Rosa ${ }^{\mathrm{e}}$, V.J. Sánchez-González ${ }^{\mathrm{a}}$, E.D. Arias-Merino ${ }^{\mathrm{e}}$ and \\ I.E. Velázquez-Brizuela ${ }^{a}$ \\ ${ }^{a}$ Laboratorio Desarrollo-Envejecimiento, Enfermedades Neurodegenerativas, Division Neurociencias, Centro de \\ Investigación Biomédica de Occidente (CIBO), Instituto Mexicano de Seguro Social (IMSS), Guadalajara, Jalisco, \\ México \\ ${ }^{\mathrm{b}}$ Centro Universitario de Ciencias Exactas e Ingeniería (CUCEI), Universidad de Guadalajara, Guadalajara, \\ Jalisco, México \\ ${ }^{\mathrm{c}}$ Departamento de Bioquímica, Instituto Nacional de Cardiología, México \\ ${ }^{\mathrm{d}}$ Departamento de Neurología, UMAE-HE-CMNO, IMSS, Guadalajara, Jalisco, México \\ ${ }^{\mathrm{e}}$ Departamento de Salud Pública, Centro Universitario de Ciencias de la Salud, Universidad de Guadalajara, \\ Guadalajara, Jalisco, México
}

\begin{abstract}
It has been suggested that mitochondrial dysfunction and defects in membrane structure could be implied in AD pathogenesis. The aim of the present work was the study of membrane fluidity in submitochondrial platelet particles and erythrocyte membranes from Mexican patients. Blood samples were obtained from 30 patients with Alzheimer disease and 30 aged-matched control subjects. Membrane fluidity determinations were done using a very low concentration of the fluorescent dipyrenylpropane probe incorporated in both types of membranes. This probe is able to give excimer and monomer fluorescence, therefore it can be used to monitor fluidity changes in biological membranes.

The data obtained showed that in submitochondrial particles from AD patients, the excimer to monomer fluorescent intensity ratio was lower $(0.231 \pm 0.008)$ than aged-matched control subjects $(0.363 \pm 0.014)$. Therefore, membrane fluidity was lower in $\mathrm{AD}$ samples. On the other hand, we found similar membrane fluidity in erythrocytes from AD patients and aged-matched controls: the fluorescent intensity ratios were $0.312 \pm 0.03$ and $0.305 \pm 0.033$, respectively. In addition, lipid peroxidation in submitochondrial particles and erythrocyte membranes was higher in AD samples than in aged-matched controls. These data suggest that submitochondrial platelet particles are more sensitive to oxidative stress than erythrocyte membranes.
\end{abstract}

Keywords: Membrane fluidity, Alzheimer disease, platelets, mitochondria, dipyrenylpropane

\footnotetext{
${ }^{*}$ Corresponding author: Genaro Gabriel Ortiz, MD, PhD, Laboratorio Desarrollo-Envejecimiento, Enfermedades Neurodegenerativas, CIBO-IMSS, Sierra Mojada 800 CP 44340, Guadalajara, Jalis-
}

co, México. Tel.: +52 333638 5593; E-mail: genarogabriel@ yahoo. com. 


\section{Introduction}

Alzheimer disease (AD) is the most common type of dementia and is characterized by progressive neurodegeneration leading to severe cognitive decline and eventual death. AD pathogenesis is yet poorly understood but is associated with extracellular neuritic plaques and intracelllular neurofibrillary tangles primarily in limbic and association cortices. Neuritic plaques are often encompassed by proliferation of activated microglia and astrocytes [27]. In addition to these neuropathological findings, it has been shown that cells of AD brains have altered phospholipid metabolism [6,11,18,25] and a diminished unesterified cholesterol:phospholipid mole ratio [21], leading to altered membrane fluidity [22]. AD brains also show reduction in pyruvate dehydrogenase [28,31], $\alpha$-ketoglurate dehydrogenase [14,20], and cytochrome c oxidase activities [23]. These defects also result in diminished ATP production, increased generation of free radicals as well as act to lower the apoptotic threshold of cells $[8,13,19]$.

Some of the biochemical abnormalities present in $\mathrm{AD}$ brains are found in other body tissues and fluids, especially in platelets, consequently it has been hypothesized that $\mathrm{AD}$ is a systemic disease [3]. In addition platelets have the following similarities with neurons: 1) contain the amyloid precursor protein and secrete $\beta$-amyloid peptide $[9,26] ; 2)$ express neurotransmitters and some neuron-related proteins, such as NMDA receptors [10].

The aim of this work is to assess the membrane fluidity in platelet submitochondrial particles and erythrocyte membranes from Mexican patients with Alzheimer disease. That estimate was achieved from the excimer to monomer fluorescence intensity ratio (Ie/Im) of the fluorescent probe 1,3 dipyrenylpropane (DPP) incorporated in membranes. That probe at submicromolar concentrations forms intramolecular excimers and its formation in membranes depends mainly of the medium microviscosity and temperature of determination. This method is very simple and does not requires higher concentrations of other fluorescent lipophilic probes such as 1,6-diphenyl-1,3,5-hexatriene (DPH) and its cationic derivative trimethylamino-DPH [30]. Thus, the risk of perturbation of the phospholipid phase inherent in the use of all probe molecules is minimized [1].

\section{Materials and methods}

\subsection{Patients}

Blood samples were obtained from 30 patients who attended the Hospital de Especialidades del Centro
Médico Nacional de Occidente at Guadalajara, Jalisco, México, with clinical suspicion of Alzheimer's disease. Informed consent was obtained from the patient and caregiver (when indicated) by a physician staff. The study was approved by the Centro de Investigaci ón Biomedica de Occidente Review board and was carried out in accordance with the principles of the Declaration of Helsinki as revised in 2000. None of the selected patients were taking antioxidants or drugs known to modify membrane fluidity.

The diagnosis of AD was made by: 1) a mini mental state evaluation (MMSE) preformed during the first visit, with a score less than 25 for an alphabetized subjects and 20 for non-alphabetized subjects; 2) DSM-IV criteria for dementia; and 3) NINCDS-ADRDA criteria for diagnosis. Patients had at least a 2.5-year history of cognitive decline. Control subjects were recruited according to the following: 1) a MMSE score of $\geqslant 25$ and $\geqslant 20$ for alphabetized and non-alphabetized subjects, respectively; 2) no fulfillment of DSM-IV criteria, and; 3) no family history of AD or any other dementia (family data was always confirmed by a second informant who was a first degree relative of the patient, mainly a sibling). Patients and volunteers were matched for sex and age. In the $\mathrm{AD}$ group, there were 13 women and 17 men. The mean age of the AD population was 61.2 \pm 11.8 years, whereas that of the control subjects was $64.3 \pm 9.6$ years.

\subsection{Preparation of platelet submitochondrial particles and erythrocyte membranes}

Venous blood samples from patients and matched controls were collected into tubes containing ethylenediaminetetra-acetic acid (EDTA) as anticoagulant, by a trained physician. The samples were centrifuged for 10 $\min$ at $200 \times g$ at room temperature. Erythrocytes were separated and platelet-rich plasma was then collected and centrifuged for $10 \mathrm{~min}$ at $7000 \times g$ and the pellets were suspended in $0.32 \mathrm{M}$ sucrose, $20 \mathrm{mM}$ Tris- $\mathrm{HCl}$ (pH 7.4). Submitochondrial particles from platelets were isolated according to Baracca et al. [4] and suspended in the same buffer, as above.

Erythrocytes were washed and centrifuged three times in isotonic solution ( $146 \mathrm{mM} \mathrm{NaCl}, 20 \mathrm{mM}$ HEP$\mathrm{ES}, \mathrm{pH} 7.4$ ) and then lysed by pipetting in $50 \mathrm{ml}$ ice-cold hypothonic solution buffer ( $7 \mathrm{mM} \mathrm{NaCl}, 5 \mathrm{mM}$ HEP$\mathrm{ES}, \mathrm{pH} 7.4,0.1 \mathrm{mM}$ phenylmethylsulfonyl fluoride) and centrifuged at $12000 \mathrm{xg}$ for $10 \mathrm{~min}$ and washed three times to remove hemoglobin. White ghosts were resuspended in $100 \mu \mathrm{L}$ of $5 \mathrm{mM}$ HEPES and resealed by incubation at $37^{\circ} \mathrm{C}$ for $1 \mathrm{hr}$. 


\subsection{Membrane phospholipid determination}

Membrane lipids from platelet submitochondrial particles and erythrocytes were extracted by the method described by Bligh and Dyer [5]. Phospholipids were separated by thin-layer chromatography [30] and scraped off the plates and digested for the assessment of phosphorus content as described by Sumner [29].

\subsection{Membrane fluidity determinations}

Membrane fluidity determinations were done with the DPP fluorescent probe in the following medium: $10 \mathrm{mM}$ Tris- $\mathrm{HCl}$ buffer ( $\mathrm{pH} 7.8)$ and $0.2 \mathrm{mM}$ membrane phospholipids (from platelet submitochondrial particles or erythrocytes). DPP solution in ethanol of spectroscopic grade was diluted and mixed with membranes given a molar ratio of fluorescent probe to membrane phospholipids of 1:1500. Alternatively, $0.25 \mathrm{mg}$ of mitochondrial protein and $0.1 \mathrm{nmol} \mathrm{DPP}$ were mixed and diluted with the same buffer as above. The mixtures were incubated in darkness at $4{ }^{\circ} \mathrm{C}$ for 5 hours, in order to achieve maximal incorporation of the fluorescent probe to the membranes. Fluorescence of DPP incorporated into membranes was measured at $24^{\circ} \mathrm{C}$ on a Perkin Elmer fluorescence spectrometer, LS50B. The fluorophore was excited at $329 \mathrm{~nm}$ and the monomer and excimer fluorescence intensities were read at 379 and $480 \mathrm{~nm}$, respectively. From these readings, the excimer to monomer fluorescence intensity ratio $(\mathrm{Ie} / \mathrm{Im})$ was calculated. Fluorescence corrections obtained from readings of membranes without DPP were applied to all fluorescence values. In initial experiments, emission fluorescence spectra were recorded, with the excitation wavelength as above.

\subsection{Lipid peroxidation}

Submitochondrial particles from platelets and white ghosts from erythrocytes were tested for lipid peroxidation using a Kit from Oxford Biomedical Res. Inc. (Product No. FR12). Each measurement was repeated twice. The Kit contains a chromogenic reagent which reacts with the lipid peroxidation products malonaldehyde (MDA) and 4-hydroxyalkenals (4-OHA) at $45^{\circ} \mathrm{C}$ yielding a stable chromophore with maximal absorbance at a wavelenght of $586 \mathrm{~nm}$. The light wavelength and low incubation temperature used for measurements eliminated interference and undesirable artifacts. Results are expressed as nmol MDA+4-OHA $(\mathrm{mg} \text { protein })^{-1}$.

Protein concentration was determined by the Bradford method using bovine serum albumin as a standard [7].

\subsection{Statistical analysis}

Data are expressed as mean values \pm SEM. Differences in membrane fluidity or were assessed with the nonparametric Wilcoxon signed rank test for paired data. A $P$-value $<0.05$ was considered statistically significant.

\section{Results}

Figure 1 shows representative emission fluorescence spectra of DPP incorporated in platelet submitochondrial particles from a patient with $\mathrm{AD}$ and its respective control. At the same concentration of membranes and probe both spectra show absolute differences in monomer and excimer fluorescences among samples from patients and controls. Principally, the monomer emission is higher in AD patients than controls. From similar experiments the excimer to monomer fluorescent intensity ratios were calculated (Fig. 2). Although there is some variability between samples, in the patients the ratio was lower than in aged-matched control. Similar results were obtained when $0.1 \mathrm{nmol}$ DPP was diluted and mixed with $0.25 \mathrm{mg}$ of mitochondrial protein (data not shown). This suggests that the inner mitochondrial membrane in patients was less fluid than in controls. In addition, lipid oxidation was higher in AD samples than aged-matched controls (Fig. 3).

Since mature erythrocytes do not have internal membranes in contrast with platelets, we measured fluidity in erythrocyte membranes at the same fluorescent probe concentration as above. Figure 2 shows that the excimer to monomer fluorescence intensity ratio was similar in both AD and control erythrocyte membranes. Therefore, membrane fluidity in AD erythrocytes was not changed with respect to controls. Lipid oxidation in AD erythrocyte membranes was higher than control samples (Fig. 3).

\section{Discussion}

Submitochondrial particles are mainly constituted of inner mitochondrial membrane and are the site of oxidative phosphorylation and other enzymatic systems involved in the transport and utilization of metabolites. Since the importance of the inner membrane in the generation of cellular energy we evaluated its fluidity. It was done with DPP, a fluorescent probe that remains anchored predominantly between the acyl chains of fat- 


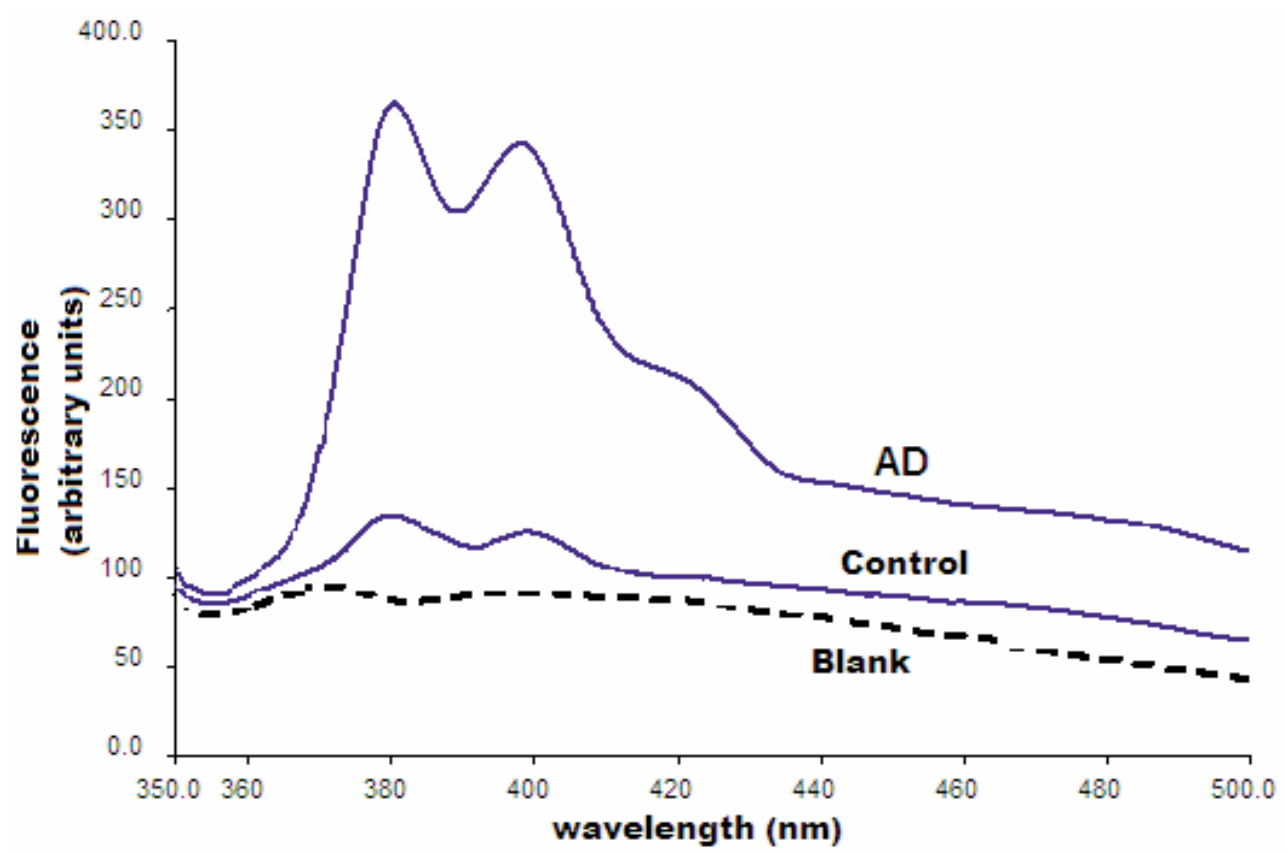

Fig. 1. Representative fluorescent emission spectra of DPP in platelet submitocondrial particles from AD patients and age-matched controls as indicated. Fluorescent probe was excited at $329 \mathrm{~nm}$. The molar ratio of membrane phospholipids to fluorescent probe was 1500 . Fluorescence emission spectra of membranes without DPP are shown (Blank).

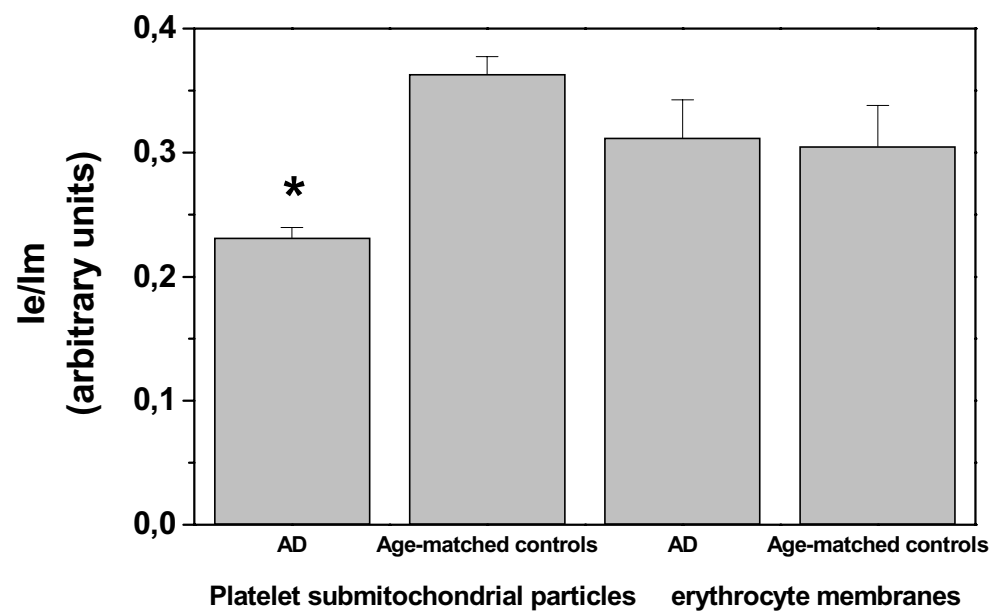

Fig. 2. Excimer to monomer fluorescent ratio of DPP on platelet submitochondrial particles and erythrocyte membranes from AD patients and aged-matched controls. The fluorescent probe was excited at $329 \mathrm{~nm}$ and emission of monomer and excimer was read at 379 and $480 \mathrm{~nm}$, respectively, as indicated in Material and Methods. The molar ratio of membrane phospholipids to fluorescent probe was 1500 . The data shown are mean \pm S.E.M. ${ }^{*} p<0.001$.

ty acids in the membrane hydrocarbon core. Because cholesterol homeostasis [21] is altered in membranes from Alzheimer disease patients we made membrane fluidity measurements using membrane phospholipid or protein concentration as normalization factor. In both conditions, the fluorescent probe was used at very low concentrations in order to minimize the alteration in membrane structure introduced by the probe itself. Intramolecular excimer formation of this probe is related with the membrane fluidity, such the excimer formation depicts mainly the lateral motion of the probe within the membrane [16]. The higher the values of 


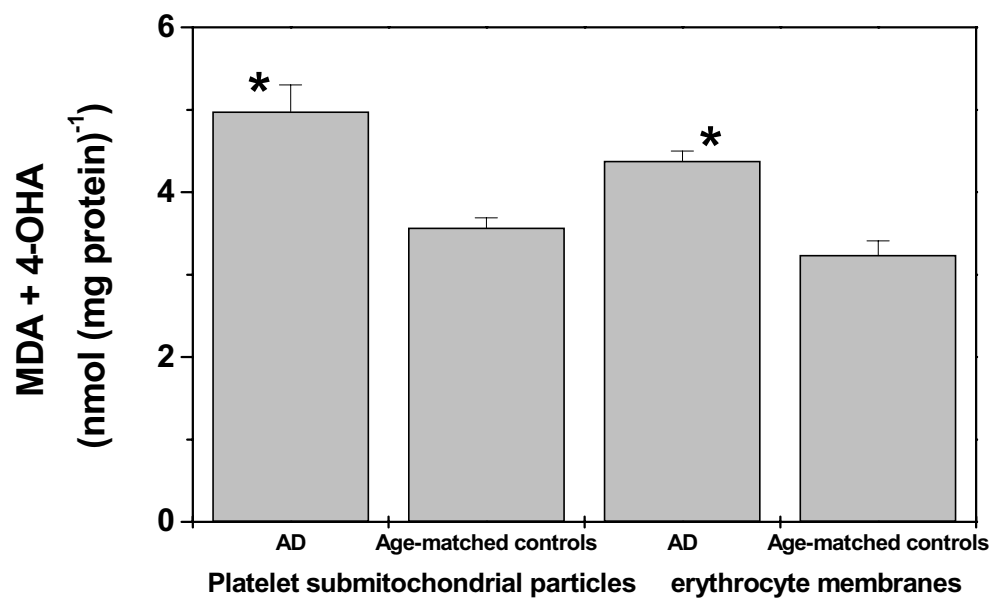

Fig. 3. Lipid oxidation products on platelet submitochondrial particles and erythrocyte membranes from AD patients and aged-matched controls. The data shown are mean \pm S.E.M. ${ }^{*} p<0.05$.

the excimer to monomer ratio, the more fluid the membrane, while the lower the ratio, the more rigid the membrane. Therefore that ratio is directly proportional to membrane fluidity, which is reciprocal to membrane viscosity.

Similarly to the data reported from mitochondria in AD brains [22], a reduced fluidity in the platelet inner mitochondrial membrane was found. It can partially be due to increased levels of lipid peroxidation.

Reduced membrane fluidity can diminish the activities of the enzymes of oxidative phosphorilation and other transport and receptor proteins, inasmuch as these enzymes are regulated by the physicochemical state of the lipid environment of the membrane. It may diminish significantly the ATP generation from the mitochondria. Interestingly, dysfunctional mitochondria and oxidative damage has been involved in Alzheimer's disease [8].

In agreement with previous reports, membrane fluidity from erythrocyte was not altered in $\mathrm{AD}$ [15], regardless of increased lipid oxidation in erythrocyte AD patients. This suggests that, in $\mathrm{AD}$, mitochondrial membranes are more sensitive to oxidative stress than erythrocytes.

In contrast to platelet inner mitochondrial membrane, it has been reported an increase in fluidity in whole membranes from platelets of AD patients [35]. This increase results from the elaboration of an internal membrane compartment resembling endoplasmic reticulum that is functionally abnormal [34]. At this regard, it is worth noting that the contribution of mitochondrial membranes to the whole cell membranes in platelets could be minimized since platelets contain few mitochondria [12].
On the other hand, Morais Cardoso et al. [24], using DPH and TMA-DPH as fluorescent probes, found similar fluidity in mitocondrial membranes in platelets from AD patients and controls. That discrepancy with our data may be due to intrinsic differences in the populations tested, the purity of the used mitochondrial fraction and the nature of the probes used.

Additionally, it's clear that the lipophilic probes are sensitive to slightly different membrane properties. For instance, DPH and TMA-DPH are rotational probes [2] and dipyrenylpropane is a lateral diffusion sensitive probe [32]. In addition, DPH partitions into the interior of the bilayer and its average location of DPH has been shown to be about $8 \AA$ from the center of the bilayer. TMA-DPH is oriented in the membrane bilayer with its positive charge localized at the lipid-water interface. Its DPH moiety is localized at about $11 \AA$ from the center of the bilayer and reports the interfacial region of the membrane [17]. Whereas dipyrenylpropane is an highly hydrophobic probe which partitions into the membrane lipid bilayer [33].

At the moment we do not know whether the diminished membrane fluidity in AD is etiologically significant or a minor result of neurodegeneration.

\section{Acknowledgments}

This work was supported by Grant 1419-5 from Fondo Sectorial en Salud-CONACyT, México. 


\section{References}

[1] L.M. Almeida, W.L. Vaz, K.A. Zachariasse et al., Fluidity of sarcoplasmic reticulum membranes investigated with dipyrenylpropane, an intramolecular excimer probe, Biochemistry 23 (1982), 5972-5977.

[2] M. Ameloot, H. Hendrickx, W. Herreman et al., Effect of orientational order on the decay of the fluorescence anisotropy in membrane suspensions. Experimental verification on unilamellar vesicles and lipid/alpha-lactalbumin complexes, Biophys J 46 (1984), 525-539.

[3] A.C. Baker, L. Ko and J.P. Blass, Systemic manifestations of Alzheimer's disease, Age 11 (1988), 60-65.

[4] A. Baracca, S. Barogi, V. Carelli et al., Catalytic activities of mitochondrial ATP synthase in patients with mitochondrial DNA T8993G mutation in the ATPase 6 gene encoding subunit a, J Biol Chem 275 (2000), 4177-4182.

[5] E.G. Bligh and W.J. Dyer, A rapid method of total lipid extraction and purification, Can J Biochem Physiol 37 (1959), 911-917.

[6] J.K. Blusztajn, I. López Gonzalez-Coviella, M. Logue et al., Levels of phospholipid catabolic intermediates, glycerophosphocholine and glycerophosphoethanolamine, are elevated in brains of Alzheimer's disease but not of Down's syndrome patients, Brain Res 536 (1990), 240-244.

[7] M.M. Bradford, A rapid and sensitive method for the quantitation of microgram quantitites of protein utilizing the principle of protein-dye binding, Analytical Biochemistry 72 (1976), 248-254.

[8] E. Bonilla, K. Tanji, M. Hirano et al., Mitochondrial involvement in Alzheimer's disease, Biochim Biophys Acta 1410 (1999), 171-182.

[9] M. Di Luca, F. Colciaghi, L. Pastorino et al., Platelets as a peripheral district where to study pathogenic mechanisms of Alzheimer disease: the case of amyloid precursor protein, Eur J Pharma 405 (2000), 277-283.

[10] C. Dreux and J.M. Launay, Blood platelets: neuronal model in psychiatric disorders, Encephale 11 (1985), 57-64.

[11] D.W. Ellison, M. Flint Beal and J.B. Martin, Phosphoethanolamine and ethanolamine are decreased in Alzheimer's disease and Huntington's disease, Brain Res 417 (1987), 389392.

[12] M.H.. Fukami and L. Salganicoff, Isolation and properties of human platelet mitochondria, Blood 42 (1973), 913-918.

[13] G.E Gibson, Interactions of oxidative stress with cellular calcium dynamics and glucose metabolism in Alzheimer's disease, Free Radic Biol Med 32 (2002), 1061-1070.

[14] G.E. Gibson, K.-F.R. Sheu, J.P. Blass et al., Reduced activities of thiamine-dependent enzymes in the brains and peripheral tissues of patients with Alzheimer's disease, Arch Neurol 45 (1988), 836-840.

[15] I. Hajimohammadreza, M.J. Brammer, S. Eagger et al., Platelet and erythrocyte membrane changes in Alzheimer's disease, Biochim Biophys Acta 1025 (1990), 208-214.

[16] A.S. Jurado, L.M. Almeida and V.C. Madeira, Fluidity of bacterial membrane lipids monitored by intramolecular excimerization of 1,3.di(2-pyrenyl)propane, Biochem Biophys Res Comm 176 (1991), 356-363.

[17] R.D. Kaiser and E. London, Location of diphenylhexatriene (DPH) and its derivatives within membranes: comparison of different fluorescence quenching analyses of membrane depth, Biochemistry 37 (1998), 8180-8190.
[18] W.E. Klunk, K. Panchalingam, R.J. McClure et al., Metabolic alterations in postmortem Alzheimer's disease brain are exaggerated by Apo-E4, Neurobiol Aging 19 (1998), 511-515.

[19] W.R. Markesbery, Oxidative stress hypothesis in Alzheimer's disease, Free Radic Biol Med 23 (1997), 134-147.

[20] F. Mastrogiacomo, C. Bergeron, S.J. Kish, Brain alphaketoglutarate dehydrogenase complex activity in Alzheimer's disease, J Neurochem 61 (1993), 2007-2014.

[21] R.P. Mason, W.J. Shoemaker, L. Shajenko, et al., Evidence for Changes in the Alzheimer's Disease Brain Cortical Membrane Structure Mediated by Cholesterol, Neurobiol Aging 13 (1992), 413-419.

[22] P. Mecocci, A. Cherubinia, M. Flint Beal et al., Altered mitochondrial membrane fluidity in AD brain, Neurosc Lett $\mathbf{2 0 7}$ (1996), 129-132.

[23] E.M. Mutisya, A.C. Bowling and M.F. Beal, Cortical cytochrome oxidase activity is reduced in Alzheimer's disease, J Neurochem 63 (1994), 2179-2184.

[24] S. Morais Cardoso, M.T. Proença, S. Santos et al., Cytochrome coxidase is decreased in Alzheimer's disease platelets, Neurobiol Aging 25 (2004), 105-110.

[25] R.M. Nitsch, J.K. Blusztajn, A.G. Pittas et al., Evidence for a membrane defect in Alzheimer disease brain, Proc Natl Acad Sci USA 89 (1992), 1671-1675.

[26] V.J. Sanchez-Gonzalez, G.G. Ortiz, P. Gallegos-Arreola et al., Altered beta-amyloid precursor protein isoforms in Mexican Alzheimer's Disease patients, Dis Markers 22 (2006), 119125.

[27] D.J. Selkoe, Translating cell biology into therapeutic advances in Alzheimer's disease, Nature 399 (1999), A23-A31.

[28] K.F.R. Sheu, Y.T. Kim, J.P. Blass et al., An immunochemical study of the pyruvate dehydrogenase deficit in Alzheimer's disease brain, Ann Neurol 17 (1985), 444-449.

[29] J.B. Sumner, A method for the colorimetric determination of phosphorus, Science 100 (1964), 413-414.

[30] J. Touchstone, J. Chen and K. Beaver, Improved separation of phospholipids in thin layer chromatography, Lipids 15 (1980), 61-62.

[31] C.M. Yates, J. Butterworth, M.C. Tennant et al., Enzyme activities in relation to $\mathrm{pH}$ and lactate in postmortem brain in Alzheimer-type and other dementias, J Neurochem 55 (1990), 1624-1630.

[32] K.A. Zachariasse, W. Kühnle and A. Weller, Intramolecular excimer fluorescence as a probe of fluidity changes and phase transitions in phosphatidylcholine bilayers, Chem Phys Lett 73 (1980), 6-11.

[33] K.A. Zachariasse, W.L.C. Vaz, C. Sotomayor et al., Investigation of human erythrocyte ghost membranes with intramolecular excimer probes, Biochim Biophys Acta 688 (1982), 323332.

[34] G.S. Zubenko, I. Malinakova and B. Chojnacki, Proliferation of internal membranes in platelets from patients with Alzheimer's disease, J Neuropathol Exp Neurol 46 (1987), $407-418$.

[35] G.S. Zubenko, U. Kopp, T. Seto et al., Platelet membrane fluidity individuals at risk for Alzheimer's disease: a comparison of results from fluorescence spectroscopy and electron spin resonance spectroscopy, Psychopharmacology 145 (1999), 175-180. 


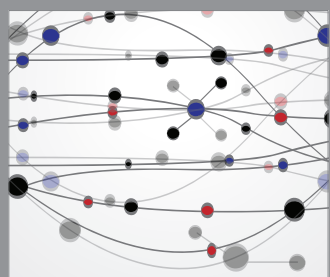

The Scientific World Journal
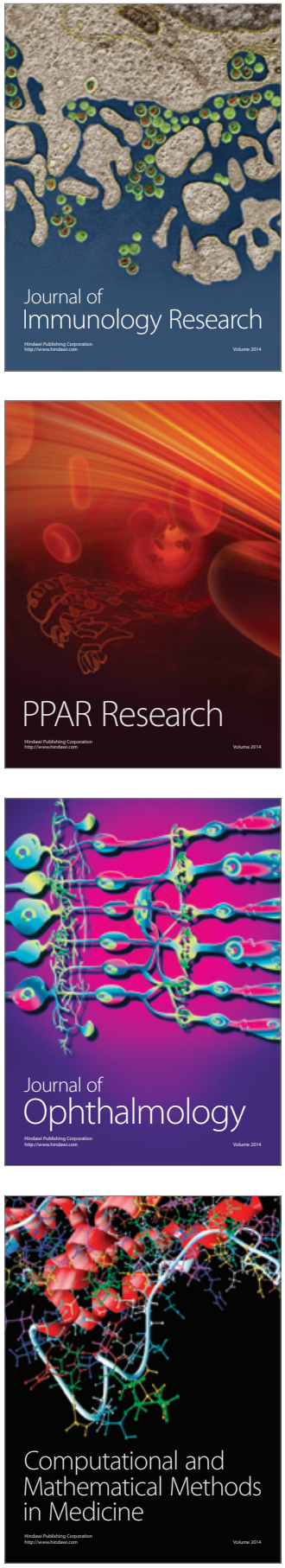

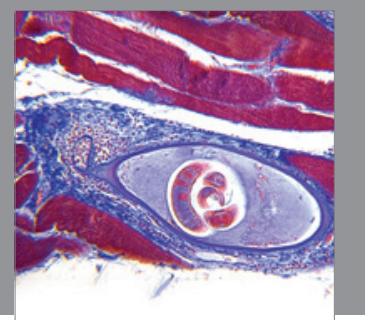

Gastroenterology

Research and Practice
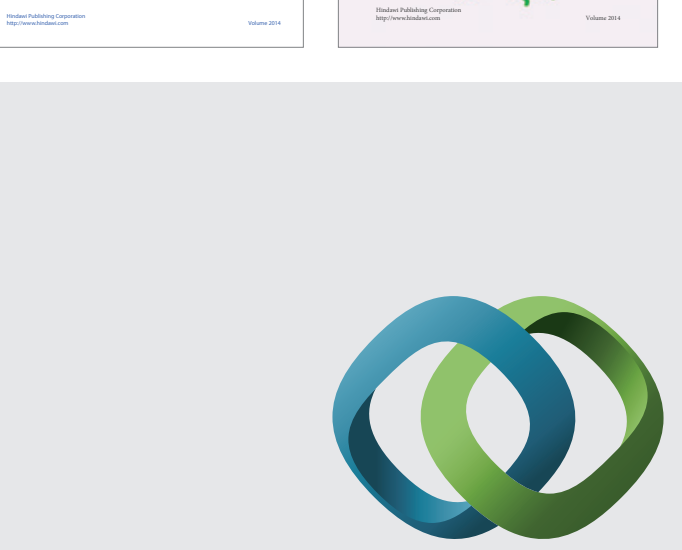

\section{Hindawi}

Submit your manuscripts at

http://www.hindawi.com
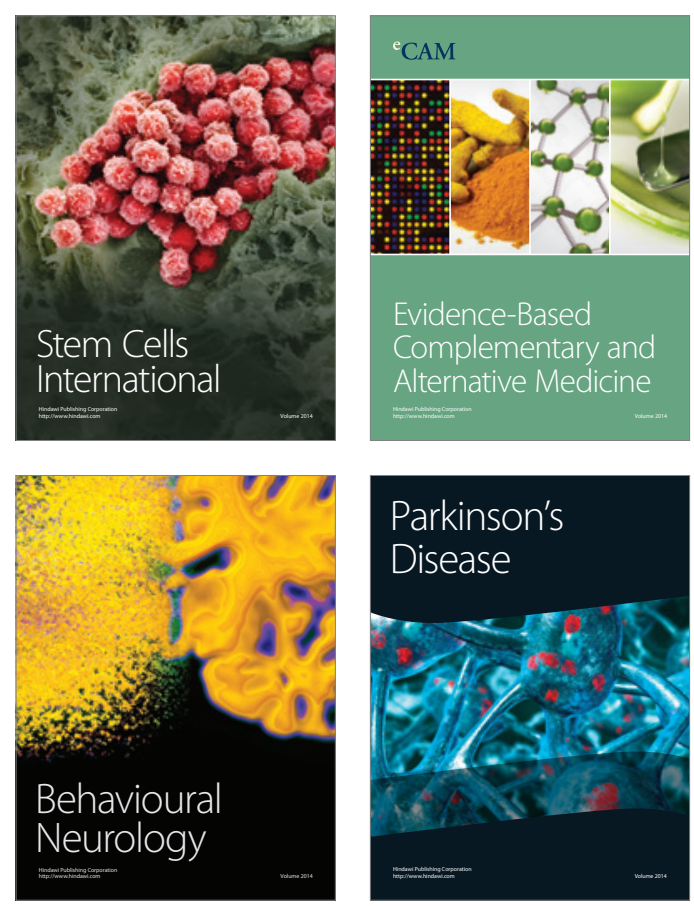

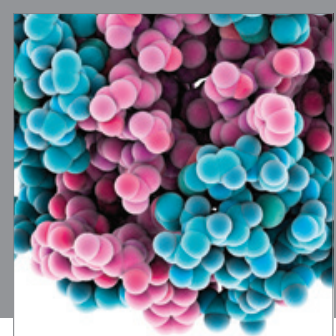

Journal of
Diabetes Research

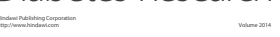

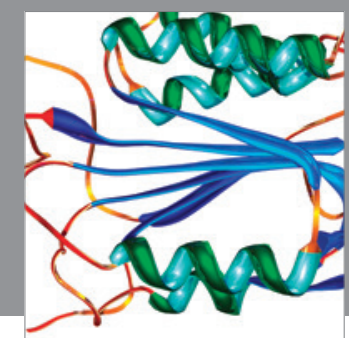

Disease Markers
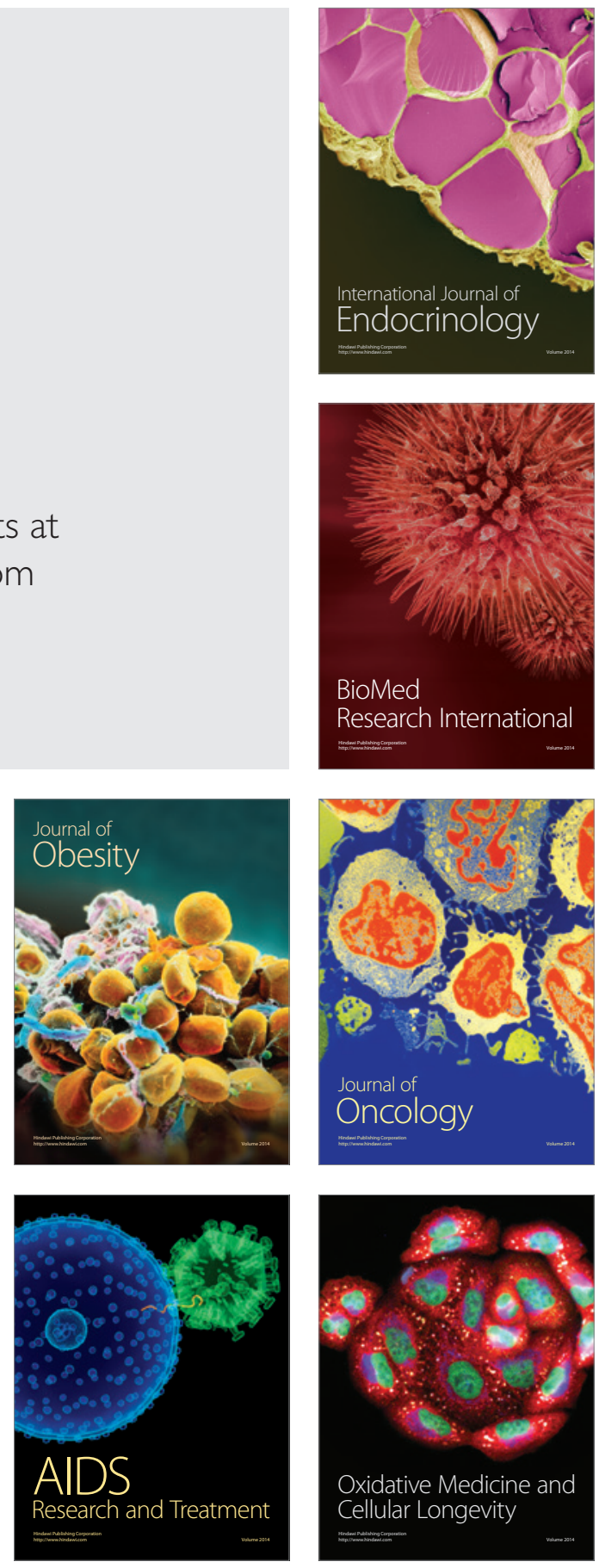